Matthias Hesse, BEd.

Allgemeine Sonderschule, Purkersdorf

\title{
COVID-19: Zur Wahrnehmung der Steuerung des Bildungssystems zu Pandemiezeiten aus der Perspektive der Sonderschulen
}

\author{
Erste Befunde einer landesweiten Befragung
}

In diesem Beitrag wird darüber berichtet, wie die (Landes-)Sonderschulen und Schulen mit angeschlossenen ASO-Klassen mit den Herausforderungen der COVID-19-Pandemie und den daraus folgenden Steuerungen des BMBWF und der Bildungsdirektionen umgegangen sind. Dafür wurden insgesamt 87 Schulen (70 Sonderschulstandorte, vier Landessonderschulen, zehn Volksschulen und drei Mittelschulen mit angeschlossenen ASO-Klassen) eingeladen, an der anonymisierten Onlineumfrage mittels LimeSurvey teilzunehmen. Von 69 Schulen $(=79,31 \%)$ wurde der Fragebogen vollständig ausgefüllt. Es handelt sich demnach um eine Vollerhebung im Bundesland Niederösterreich. Der Fragebogen wurde im Vorfeld und auf der Basis von praktischen Erfahrungsberichten und Rückmeldungen von anderen Sonderschulstandorten bzw. deren Schulleitungen erstellt und soll einen Querschnitt durch die aktuell vordergründig am meisten auftauchenden Themen darstellen.

Sonderschulen, Pandemie, Irritation, Autonomie

\section{Die COVID-19-Pandemie als Irritation für das Bildungssystem}

Die Auswirkungen der pandemischen Gesundheitskrise der vergangenen Monate haben den Alltag an Österreichs Schulen nachhaltig irritiert und stellten die scheinbare Unveränderlichkeit von Schule (Rürup, 2009) real auf die Probe. An den einzelnen Schulstandorten mussten in relativ kurzer Zeit praxistaugliche Konzepte gefunden werden, um das Lehren und Lernen weiterhin nach den gesetzlichen Vorgaben zu ermöglichen. Im Etappenplan für Schulen des BMBWF (2020, S. 11) war angeführt, dass in jedem Fall sichergestellt werden muss, dass „dem Bildungsbedarf der Kinder/Jugendlichen Rechnung getragen wird [...].“ Insbesondere für Standorte mit Schüler*innen mit sonderpädagogischem (und/oder erhöhtem) Förderbedarf ergaben und ergeben sich angesichts der Pandemie diverse 
Herausforderungen (vgl. auch Stellungnahme der Sektion Sonderpädagogik der Deutschen Gesellschaft für Erziehungswissenschaft vom 10.04.2020). Daher wurde jeder einzelne Sonderschulstandort angehalten bzw. ermächtigt, „schulautonome Lösungen zwischen Eltern, Erziehungsberechtigten und Schulleitungen sowie Pädagoginnen und Pädagogen zum Schulbesuch oder zur Ausnahme vom Schulbesuch“ zu finden (BMBWF, 2020, S. 11).

Die COVID-19-Pandemie als ursächlicher Auslöser für strukturdeterminierte Veränderungen von außen kann im systemtheoretischen Sinne nach Simon (2017, S. 53) als "Irritation“ verstanden werden. Diese „pandemischen Störungen“ haben das System Schule in zwei Instanzen irritiert:

Die primäre Irritation war die weltweite und gesamtgesellschaftliche Gesundheitskrise, die COVID-19-Pandemie selbst - mit all ihren Auswirkungen auf das Schulsystem. Die sekundäre Irritation war die Irritation der Einzelschule in Form von Gesetzen und Erlässen (z. B. Distance Learning, Testpflicht etc.). Diese Regularien und Normierungen vonseiten der Makroebene, des Bildungsministeriums bzw. der Bildungsdirektion, sollten dazu dienen, der Irritation zu begegnen und das Gleichgewicht an den einzelnen Schulstandorten wiederherzustellen.

Im vorliegenden Beitrag wird daher der Frage nachgegangen, wie die von der Makroebene gesetzten Maßnahmen von den Sonderschulen und Schulen mit angeschlossenen ASOKlassen des Bundeslandes Niederösterreich wahrgenommen bzw. umgesetzt wurden. Für folgende fünf Fragen soll im Rahmen einer quantitativ durchgeführten Onlinebefragung von Schulleiter*innen aller niederösterreichischen Sonderschulstandorten Antwort gegeben werden:

1. Wie herausfordernd erlebten die Sonderschulleiter*innen der niederösterreichischen Sonderschulen und angeschlossenen ASO-Klassen die aktuelle schulische Situation aufgrund der Pandemie?

2. Inwiefern konnte aus Sicht der Schulleiter*innen der vom BMBWF geforderte pädagogische Auftrag im Sinne eines gelingenden Lernens in der Pandemie am eigenen Schulstandort erfüllt werden?

3. Inwiefern besteht von Seiten der befragten Sonderschulleiter*innen der Wunsch, auch während der Pandemie den Präsenzunterricht beizubehalten?

4. Inwiefern ist von den Sonderschulleiter*innen gewünscht, schulautonom über die Form des Unterrichts (Präsenzunterricht, Distance Learning, Schichtbetrieb etc.) entscheiden zu dürfen?

5. Wie gelingend wurde die Umsetzung der Hygiene- und Präventionsmaßnahmen und der Testungen eingeschätzt?

Die Untersuchungsfragen wurden (online) mittels Befragung von Schulleiter*innen aus der allgemeinen Sonderschule und Schulen mit ASO-Klassen beantwortet. Die Befragung fand im März 2021 anonymisiert via LimeSurvey statt. Von den gesamt 87 Schulleiter*innen nahmen 69 an der Befragung teil. Limitierend ist anzuführen, dass es sich bei den Antworten um die subjektive Sichtweise der Befragten handelt. 


\section{\# schuleverantworten}

führungskultur_innovation_autonomie

Die Datenanalyse erfolgte mit SPSS und Excel. Die Auswertungen werden in einem weiteren Schritt auf deskriptiver Ebene dargestellt.

\section{Ergebnisdarstellung}

In einem ersten Schritt sollte erfasst werden, in welchem Ausmaß die befragten Schulen von COVID-19-Erkrankungen betroffen waren (vgl. Abbildung 1).

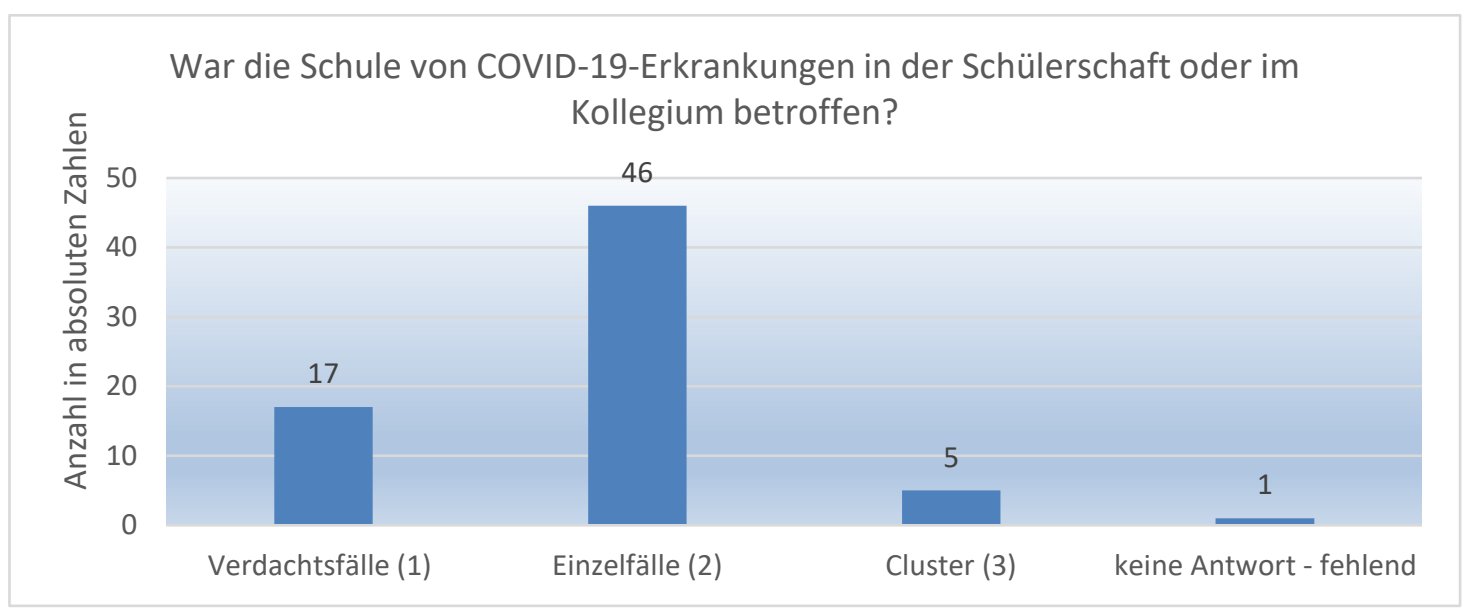

Abbildung 1: Betroffenheit der Schulen mit COVID-19-Erkrankungen.

Von den 69 befragten Schulleiter*innen $(=100 \%)$ gaben $46(=66,7 \%)$ an, von einzelnen Fällen an COVID-19-Erkrankungen (unter 10\%) betroffen gewesen zu sein. Weitere $5(=7,2 \%)$ hatten einen Cluster (über 10\%) an der Schule. Verdachtsfälle meldeten 17 Schulleiter*innen $(=24,6 \%)$. Eine Person entzog sich der Antwort.

Ferner interessierte, wie herausfordernd die Schulleiter*innen der niederösterreichischen Sonderschulen und angeschlossenen ASO-Klassen die aktuelle schulische Situation aufgrund der COVID-19-Pandemie erlebten. Die Auswertung ergab, dass die Pandemie von 55 (= 80,9\%) der befragten Schulleiter*innen als Herausforderung („trifft zu“ und "trifft eher zu“) wahrgenommen wurde (vgl. Abbildung 2), diese jedoch im Vergleich zu den anderen Schulformen als ausgewogen bzw. vergleichbar eingeschätzt wurde. 


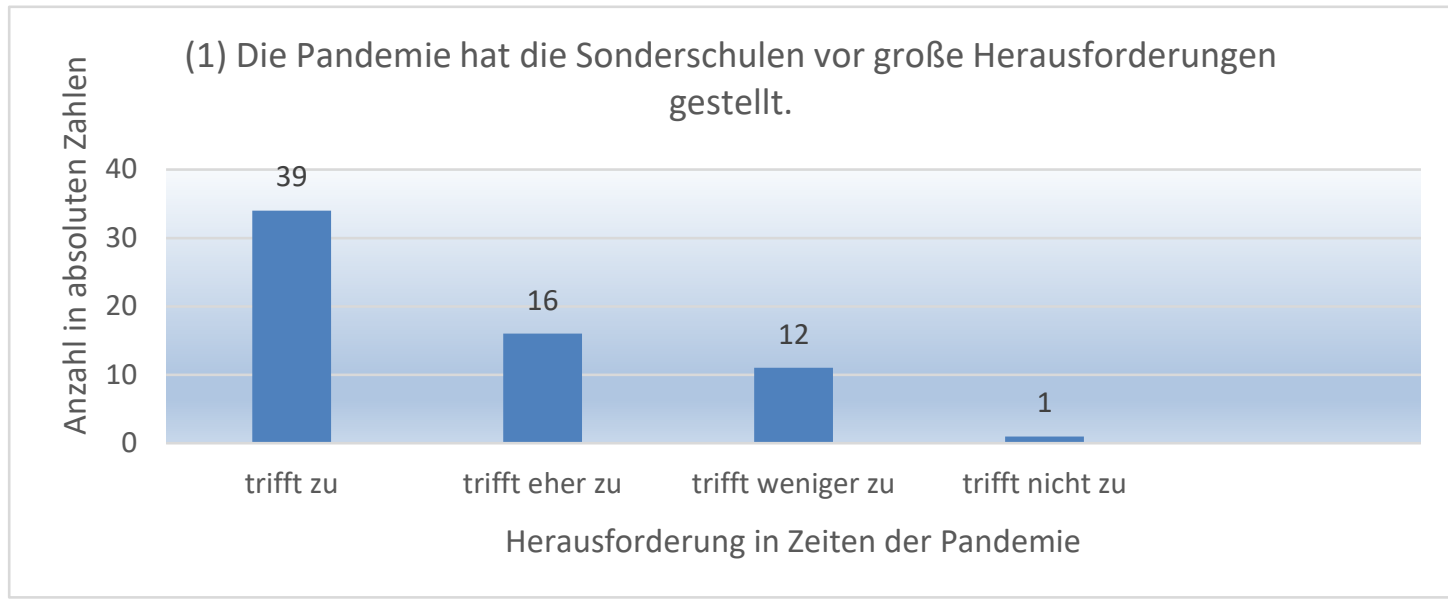

Abbildung 2: Einschätzung der Herausforderungen der Schulen in Zeiten der Pandemie

In einer weiteren Frage ging es um die Umsetzung des vom Bundesministerium geforderten pädagogischen Auftrages im Sinne eines gelingenden Lernens während der Pandemie. Die Ergebnisse zeigen, dass 63 (=91,3\%) der 69 teilnehmenden Schulleiter*innen angaben, den Bildungsauftrag im Sinne der Normierungen bzw. der Lehrpläne erfüllt zu haben („trifft zu“und „trifft eher zu“). Die Umsetzung als weniger gut gelungen schätzten $6(=8,7 \%)$ der befragten Personen ein.

Die Frage zur Unterrichtsorganisation und Unterrichtsform in Bezug auf Präsenzunterricht, Distance Learning, Schichtbetrieb etc. ließen eine klare Tendenz dafür erkennen, dass die Sonderschulen vorzugsweise im Präsenzunterricht geführt werden sollten. So gaben $32(=46,4 \%)$ der befragten Sonderschulleiter*innen eine eindeutige Präferenz („,trifft zu) und 22 (= 31,9\%) eine tendenzielle Zustimmung (,trifft eher zu) für diese Unterrichtsform an. Ablehnend („trifft weniger" und "trifft nicht zu“) zeigten sich 13 (= 18,8\%) Schulleiter*innen. Lediglich zwei Personen $(=2,9 \%)$ entzogen sich der Antwort.

Eine eindeutige Aussage konnte bei der Frage erzielt werden, ob die Sonderschulen autonom über die Form des Unterrichts (Präsenzunterricht, Distance Learning, Schichtbetrieb etc.) entscheiden sollten. Hier ordneten sich $63(=92,6 \%)$ der 69 (= 100\%) Standorte bei der eindeutigen Zustimmung in der Skalierung "trifft zu“ und "trifft eher zu“ ein.

Die Umsetzung und Praxistauglichkeit der Hygiene- und Präventionshandbücher, bzw. die dazugehörigen Verordnungen und Erlässe konnten von einer klaren Mehrheit, $d$. h. von 54 (= 78,3\%, 37,7\% „trifft zu“, 40,6\% „trifft eher zu“) Schulleiter*innen mit den Realitäten an Sonderschulen in Einklang gebracht werden. Als "weniger" und "nicht gelungen“ schätzten $15(=21,7 \%)$ der Befragten deren Umsetzung und Praxistauglichkeit ein. 


\section{\# schuleverantworten}

Eindeutig zeigt sich die Qualitätseinschätzung der Maßnahmeneffizienz beim Thema "Testung“. So geben $100 \%$ (=69) der befragten Schulleiter*innen an („trifft zu“und „trifft eher zu“), dass die Tests den Umständen entsprechend funktioniert hätten.

Interessiert hat ferner, ob es einen erkennbaren Zusammenhang zwischen Anzahl der COVID19-Erkrankungen am Schulstandort und der erlebten Herausforderung in der Pandemie gibt.

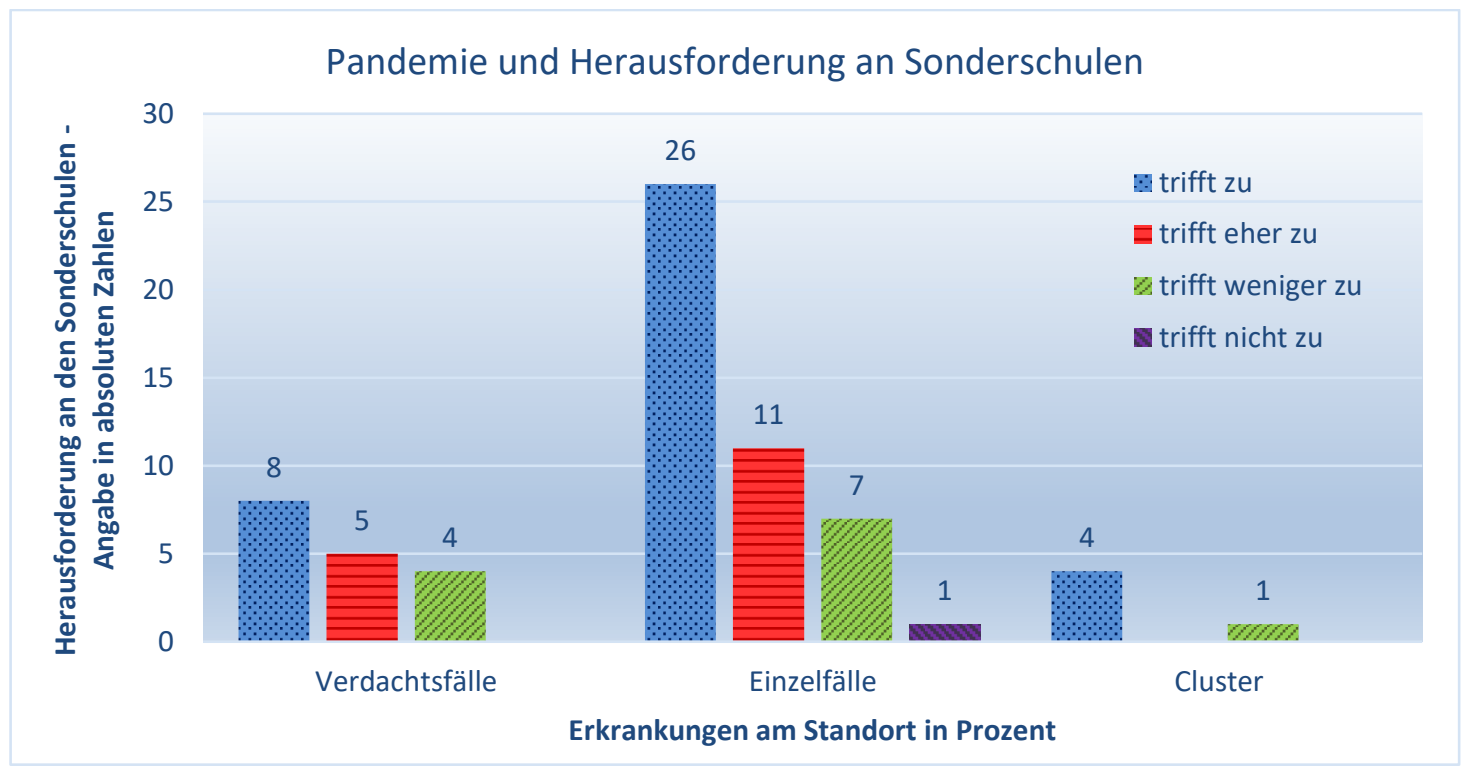

Abbildung 3: Zum Zusammenhang der Erkrankungen und der Herausforderungen am Standort $(n=69$; 2 missings)

Da es sich um eine asymmetrische Fragestellung handelt, wurde der Zusammenhang mittels Kreuztabelle im Sinne von Zeisel (zit. nach Kast, 2016, S. 91 f) errechnet. Das Ergebnis zeigt mit $r=0,063$, dass kein Zusammenhang zwischen der Anzahl der COVID-19-Fälle am eigenen Schulstandort und dem Erleben der Herausforderung in Zusammenhang mit der Pandemie vorliegt (siehe Abbildung 3). 


\section{\# schuleverantworten}

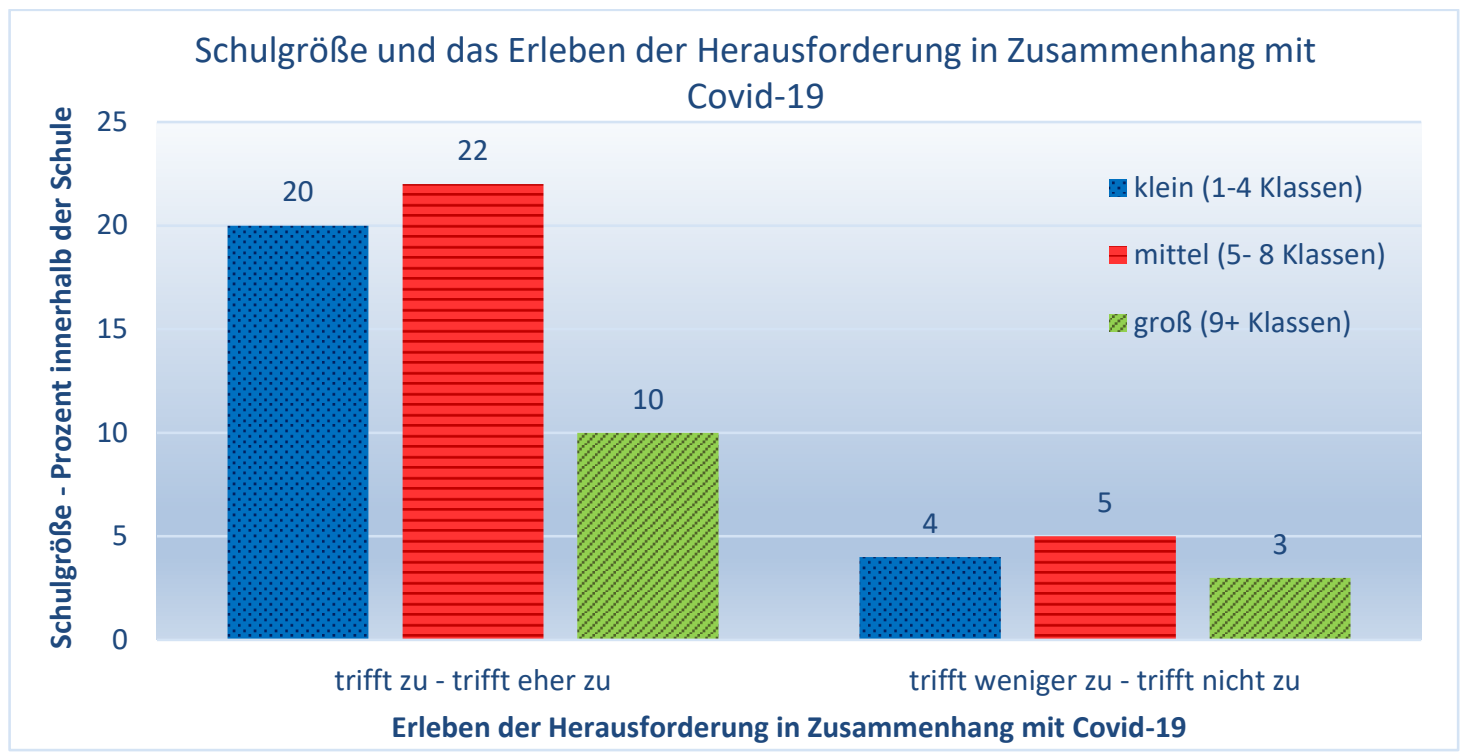

Abbildung 4: Zum Zusammenhang der Schulgröße und der Herausforderung am Standort ( $\mathrm{n}=69 ; 5$ missings)

Abbildung 4 zeigt weiters, dass auch die Schulgröße im Vergleich mit den COVID-19Erkrankungen eine untergeordnete Rolle spielt. Es ließ sich kein Zusammenhang zwischen der Schulgröße und dem Erleben der Herausforderung in Bezug auf die COVID-19-Pademie $(r=0,06)$ erkennen.

\section{Zusammenfassung und Ausblick}

Die Intention des vorliegenden Artikels war es, einen Beitrag bei der Klärung der Frage zu schaffen, wie die Steuerung des Bildungssystems in Pandemiezeiten aus der Sicht der Sonderschulen wahrgenommen wurde.

Auf der Basis der hier vorliegenden Befunde wird deutlich, dass der bisherige Verlauf der Pandemie und den gesetzten Maßnahmen von Seiten der Makroebene bzw. der Bildungsdirektion eine große Herausforderung für die befragten Standorte darstellt. Es kann jedoch festgehalten werden, dass die Sonderschulleiter*innen diese Herausforderung im Vergleich mit anderen Schulformen verhältnismäßig analog einschätzen.

Mit der Verwendung schulautonomer Konzepte (vgl. „Suchbewegung der Sonderschulen nach Homöostase") konnte der Schulalltag an den Standorten laut der vorliegenden Auswertung und Einschätzung der Schulleiter*innen gut organisiert und den neuen Herausforderungen entsprechend adaptiert werden. Der systemische Versuch, Stabilität zu erreichen und "Normalität" wiederzuerlangen, dürfte im kleinsten gemeinsamen Nenner dahingehend geglückt sein, den Schüler*innen trotz der Pandemie und stets den Umständen entsprechend einen lernwirksamen Unterricht zu ermöglichen. 


\section{\# schuleverantworten}

führungskultur_innovation_autonomie

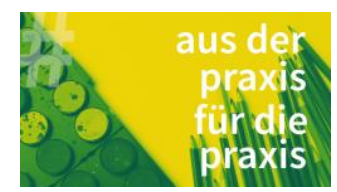

Zusammenfassend kann festgehalten werden: Die Steuerung des Bildungssystems erreicht die Standorte in Form der Maßnahmen und wird nach Adaption und dem Befinden der Schulen entsprechend mehrheitlich umsetzbar und wirksam beschrieben. Die intrinsische Kompetenz, von sich aus auf diese Herausforderung mit kurativen Ansätzen von innengesteuerten Verhaltensweisen in der Organisation Schule zu reagieren, bleibt ein Wesensmerkmal der Sonderschulen und komplettiert - zusammen mit dem Wunsch nach Autonomie - das Bild im Umgang mit der Pandemie.

\section{Literaturverzeichnis:}

BMBWF: Bundesministerium für Bildung, Wissenschaft und Forschung (2020). Etappenplan zur Aktivierung des Schulbetriebs. Abgerufen am 01.04.2021 unter https://www.bmbwf.gv.at/Themen/schule/beratung/corona/corona info/corona etappenplan. $\underline{\mathrm{html}}$

DGFE: Deutsche Gesellschaft für Erziehungswissenschaft (10.04.2020). Stellungnahme der Sektion Sonderpädagogik. Abgerufen am 01.04.2021 unter https://www.dgfe.de/sektionenkommissionen-ag/sektion-6-sonderpaedagogik/stellungnahmen

Fend, H. (2008). Schule gestalten-Systemsteuerung, Schulentwicklung und Unterrichtsqualität. Wiesbaden: VS Verlag für Sozialwissenschaften.

Herrmann, P. (2014). Einführung in das systemische Schulmanagement. Heidelberg: Carl-Auer Verlag.

Kast, F. (2016). Analyse kategorialer Daten mit Kontingenztabellen. In H. Schwetz, R. Beer, I. Benischek \& A. Forstner-Ebhart (Hrsg.), Einführung in das quantitativ orientierte Forschen und ersten Analysen mit SPSS (4. Aufl.; S. 87-95). Wien: Facultas.

Reisinger, M. (2016). Was leisten Effektgrößen. In H. Schwetz, R. Beer, I. Benischek \& A. Forstner-Ebhart (Hrsg.), Einführung in das quantitativ orientierte Forschen und ersten Analysen mit SPSS (4. Aufl.; S. 169 -182). Wien: Facultas.

Rost, D. H. \& Sparfeldt, J. (Hrsg.) (2018). Handwörterbuch Pädagogische Psychologie (5. Aufl.). Weinheim/Basel: Beltz Verlagsgruppe.

Rürup, M. (Hrsg.) (2009). Invarianzen der Schulorganisation. Wuppertal: Bergische Universität Wuppertal.

Simon, F. (2017). Einführung in Systemtheorie und Konstruktivismus. (8. Aufl.). Heidelberg: Carl-Auer Verlag. 


\section{Autor*innen}

\section{Matthias Hesse, BEd.}

Schulleiter der Allgemeinen Sonderschule Purkersdorf, in der Fortbildung Fach- und Diplomausbildung - Behindertenarbeit und Pflegeassistenz für Unterstützte Kommunikation und Sonderpädagogik tätig, Schülerberater, Layout und Producing, Grafik- und Kommunikationsdesign.

Kontakt: aso.purkersdorf@noeschule.at 